(C) 2018. This manuscript version is made available under the CC-BY-NC-ND 4.0 license http://creativecommons.org/licenses/by-nc-nd/4.0/

\title{
Micrometric non-contact position magnetoimpedance sensor
}

\author{
J.J. Beato-López ${ }^{1,2}$, I. Royo-Silvestre ${ }^{1}$ and C. Gómez-Polo ${ }^{1,2}$ \\ ${ }^{1}$ Depto. de Ciencias. Universidad Pública de Navarra, Campus de Arrosadia, 31006 \\ Pamplona, Spain \\ ${ }^{2}$ Institute for Advanced Materials (INAMAT), Universidad Pública de Navarra, Campus \\ de Arrosadia, 31006, Pamplona, Spain.
}

Abstract.- In this work a sensitive micrometric non-contact position sensor based on the Giant MagnetoImpedance effect (GMI) is analyzed. A nearly zero magnetostrictive $\mathrm{CoFeSiBCr}$ wire was employed as sensor nucleus. The sensing principle is based on the changes in the high frequency electric impedance, $Z$, of the soft magnetic element as a function of the relative position of a permanent magnet generating a non-uniform magnetic field along the wire's axis. The sensor sensitivity is analyzed in terms of the magnetic field gradient and wire's length. The comparison between the sensing response of a single wire element and a long wire $(12 \mathrm{~cm}$ in length) with different voltage contacts along its axis is performed. Higher micrometric sensitivities are achieved in wires with a certain critical length. A slight enhancement of the sensor sensitivity is found under the single wire configuration below the critical wire length. These results are interpreted as the contribution of the characteristic closure domain structure at the sample ends in these soft magnetic wires. Finally, the application of the sensor for the detection of the daily micrometric trunk shrinkage variations in a lemon tree is presented. The results indicate that this type of magnetic sensors can be easily implemented in the agricultural sector, providing a low cost and sensitive detection technique regarding water monitoring purposes.

\section{Corresponding autor.-}

Prof. Cristina Gómez-Polo

Departamento de Ciencias. Edificio de los Acebos.

Universidad Pública de Navarra. Campus de Arrosadia. 31006 Pamplona. Spain Phone. +34-948169576/Fax: +34-948169565/e.mail: gpolo@unavarra.es

Keywords.- Giant magnetoimpedance; magnetic sensors; soft magnetic amorphous wires. 


\section{1.- Introduction}

Magnetic sensors are widely employed in different technological sectors [1-3], including magnetometers for the determination of the magnetic field and devices employed to characterize associated physical magnitudes as displacement, electric current, temperature, or strain/stress. In particular, position detectors represent a sensor family with broad economic and technological significance. In fact, different market studies show that the position sensor market is expected to continue increasing in the next years (i.e. USD 5.98 Billion by 2022 [4]). Such a continuous demand is mainly driven by the development of smart electronic systems and robotics for different industrial sectors such as industrial manufacturing or automotive industry. Generally, position sensors can be classified according to the contact or non-contact operation principle. Although contact sensors (i.e. resistive potentiometers) display cost advantages, non-contact technology is usually preferred due to the longer durability and higher accuracy. Regarding magnetic technology, the simplest non-contact position device is based on the measurement of the magnetic field generated by a permanent magnet by sensitive magnetometers as a function of the relative position. Thus, Hall-effect and magnetoresistance (AMR) magnetic sensors are nowadays widely commercially employed, both under linear and rotary configurations.

On the other hand, the so-called Giant Magnetoimpedance (GMI) effect has been shown as an alternative technique in the development of highly sensitive low cost magnetic sensors [5-7]. This effect consists in the detection of huge changes in the high frequency electric impedance, $Z$, under the action of an external applied magnetic field, $H$ [8]. Within the framework of classical electrodynamics, $\mathrm{Z}$ can be expressed as [9]:

$$
Z=\left(\frac{L}{2 \pi \sigma a}\right) k \frac{J_{0}(k a)}{J_{1}(k a)}
$$


with $a$ : wire radius; $\sigma$ : electric conductivity; $k=\frac{1-j}{\delta}, \delta=\sqrt{\frac{1}{\pi f \sigma \mu}} ; \mu$ : magnetic permeability; and $J_{i}(k a)$ the Bessel functions of first kind. Thus, in the high frequency range $Z$ would mainly be determined by the skin effect and the application of an external $H$ field would promote measurable variations in $Z$ as a consequence of the changes in $\mu$. Since the discovery of the physical phenomenon in the mid-90s $[10,11]$ different sensor configurations have been analyzed $[12,13]$. In particular, non-contact position GMI technology has been also proposed in the literature [14-16]. However, practical implementations and the analysis of the optimum sensing conditions have been scarcely reported. In this work, a non-contact position sensor is analyzed based on the Giant MagnetoImpedance effect (GMI) employing a nearly zero magnetostrictive FeCo soft magnetic wire and a ferrite permanent magnet. The sensitivity optimization is analyzed in terms of the magnetic field gradient associated to the permanent magnet and the effective wire length. Micrometric sensitivity is achieved enabling the design of highly sensitive non-contact position sensors. In particular, the proposed non-contact sensing technology is applied to monitor the micrometric variations of the mean diameter of the trunk in plants. Measurement of trunk diameter variations has been proposed as the most efficient technique for irrigation scheduling in the agricultural sector [17, 18]. During daylight, the trunk shrinks as a result of water loss due to evaporation while an opposite evolution (increase in the trunk mean diameter) is found at night as a result of withinplant water balance. Monitoring this daily trunk evolution provides valuable information about the water plant status and thus enables efficient irrigation scheduling. The proposed non-contact magnetic technology displays comparable micrometric sensitivity than a commercial Linear Variable Differential Transducer (LVDT) for monitoring the trunk diameter variations in a young lemon tree. This low cost sensing technique could provide sustainable sensing technology for the agricultural sector, not only regarding 
environmental purposes (protection of water resources) but also in the improvement of crop productivity and quality.

\section{2.- Experimental procedure}
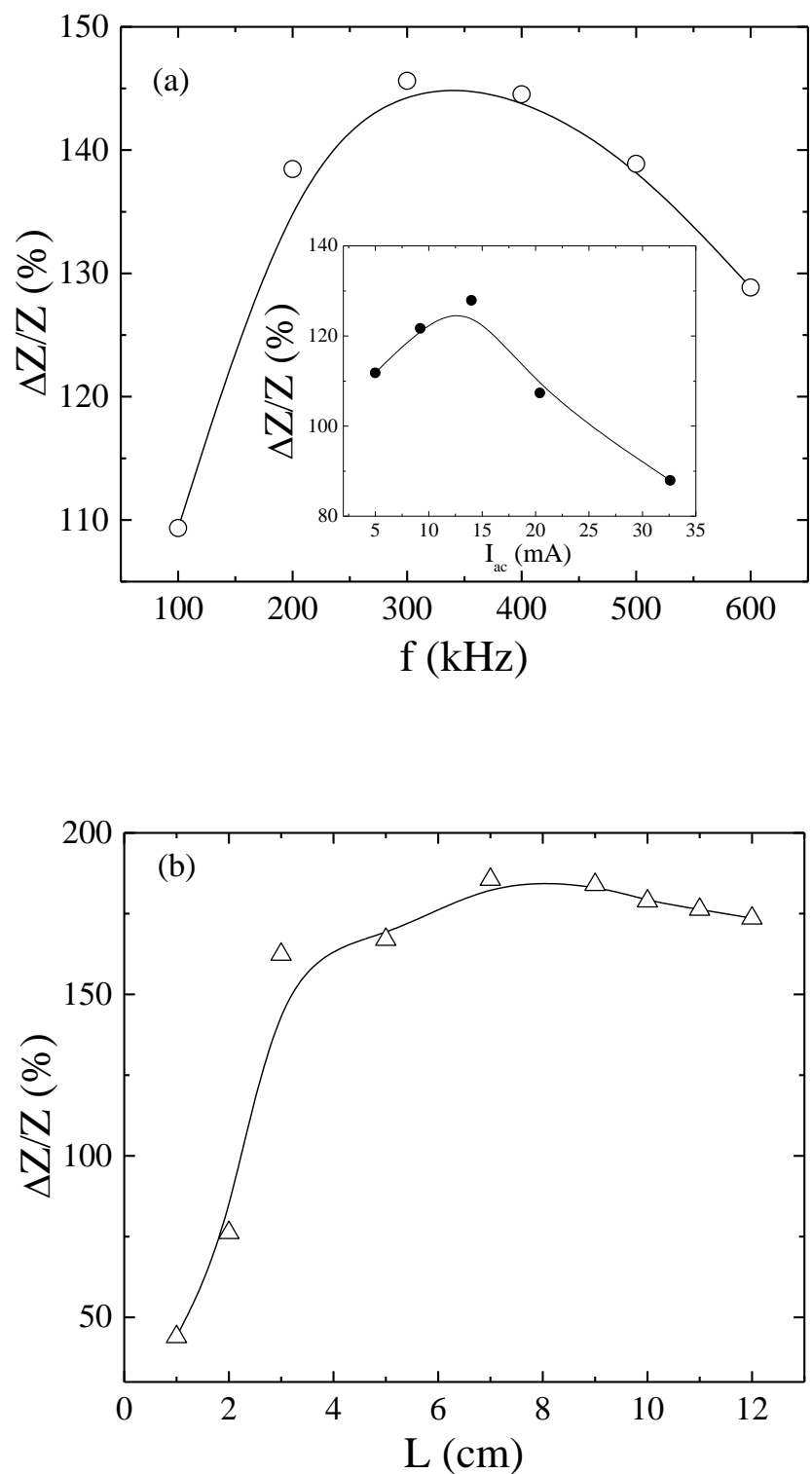

Fig. 1: (a) GMI ratio as a function of the exciting frequency, $f$, (current amplitude, $I_{p p}=$ $15 \mathrm{~mA}$ ) and versus $I_{p p}$ (inset) for a wire $3 \mathrm{~cm}$ in length. (b) GMI ratio as a function of the sample length, $L$ (frequency $f=300 \mathrm{kHz}$ and current amplitude, $I_{p p}=15 \mathrm{~mA}$ ). 
The wire was excited with an $a c$ current, $I$, frequency, $f$, and peak-to peak amplitude, $I_{p p}$. The output voltage $(V=Z I)$ measured under voltage-divider configuration, was detected through a Hewlett Packard 34401A multimeter, being the whole system controlled by LABVIEW 2014. Optimum current conditions were determined through the maximum magnetoimpedance changes under a $d c$ axial uniform magnetic field, $H$, generated by a home-made solenoid. Fig.1a shows the evolution of the GMI ratio defined as $\frac{\Delta Z}{Z}(\%)=\left(\frac{Z(H=0)-Z(\operatorname{Hax}))}{Z(\operatorname{Hax})}\right) \times 100\left(H_{\max }=4.4 \mathrm{kA} / \mathrm{m}\right)$ as a function of the frequency, $f$, and current amplitude, $I_{p p}$, for a wire $3 \mathrm{~cm}$ in length. Maximum GMI ratios are obtained for $f=300 \mathrm{kHz}$ and $I_{p p}=15 \mathrm{~mA}$. Fig. $1 \mathrm{~b}$ shows the dependence of the GMI ratio as a function of the wire length under the selected current conditions. A clear increase in $\frac{\Delta Z}{Z}(\%)$ with $L$ is observed, displaying a slight maximum around $7 \mathrm{~cm}$.

The non-contact position detection is based on the changes of the wires`s impedance as a function of the relative position, $x$, of a permanent magnet generating a magnetic field, $H_{m}$, along the sensor axis (see fig. 2). Initially, the effect of the magnetic field gradient on the sensor response was analyzed in a wire $3 \mathrm{~cm}$ in length employing two commercial ferrite permanent magnets: (i) rectangular $2 \mathrm{~cm} \times 1 \mathrm{~cm} \times 0.5 \mathrm{~cm}$ and (ii) cylindrical with diameter $5 \mathrm{~mm}$ and height $5 \mathrm{~mm}$. The magnetic field gradient $\left(H_{m}\right.$ versus $x$ ) was measured through a Lakeshore 425 gaussmeter. Regarding the micrometric characterization a micrometric screw with $10 \mu \mathrm{m}$ step was employed to suitably control the position of the rectangular permanent magnet. The sensor output, $V(x)$, was characterized for wires as a function of the wire length $L(1 \mathrm{~cm} \leq L \leq 12 \mathrm{~cm})$. The achieved micrometric sensitivity is compared with that obtained in a different configuration denoted multicontact. A single $12 \mathrm{~cm}$ length wire was excited with an $a c$ current between the sample ends ( $A-C$ contacts, see fig 2$)$ and the voltage drop across 
different segments of wire ( $A-B$ contacts) was measured. Thus, effective segment length, $L_{c}$, were chosen modifying the $A-B$ distance (see fig. 2).

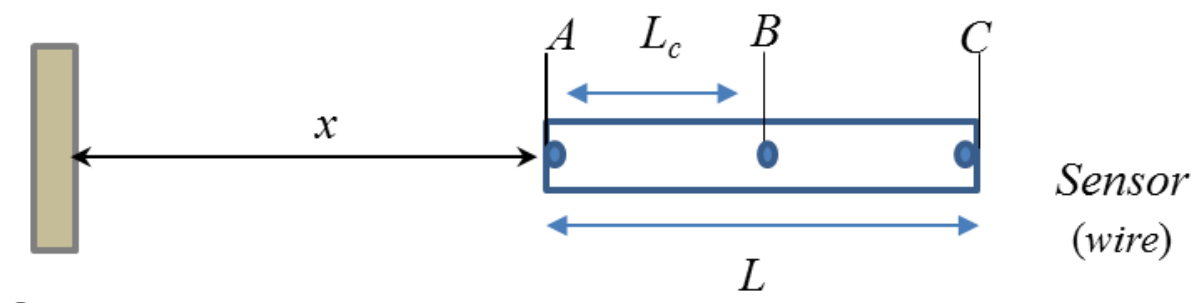

\section{Magnet}

Fig. 2: Sensor configuration schedule

In order to demonstrate the applicability of this sensing technology in agronomical environment, the sensor was attached to a young lemon tree. The rectangular magnet was glued to the main plant trunk and the sensing wire was attached to the lemon tree's employing an invar support to avoid any change in the relative distance between the magnet and wire $(x)$ due to dilatation effects. A fitotron growth chamber was employed in the sensor characterization, where the periods of fluorescent illumination and darkness were periodically changed every 12 hours. The temperature was kept constant within the temperature chamber control around $21{ }^{\circ} \mathrm{C}$. A commercial LVDT sensor was also attached to the lemon tree to simultaneously measure the variations in the trunk. These happen as a result of the water loss due to evaporation occurring during the illumination period, gives rise to an increase in the effective distance magnet-wire, $x$. Conversely, the trunk growth during the darkness period (within-plant water balance) has associated a decrease in $x$. 


\section{Results and discussion}

\subsection{Sensor characterization: effect of the magnetic field gradient}

Fig. 3a shows the sensor response (output voltage, $V$, versus the relative magnet position, $x$ ) for the two analyzed ferrite permanent magnets $(L=3 \mathrm{~cm})$. It is clearly shown the dependence of the sensor response (sensor span and sensitivity) on the employed permanent magnet. In the same figure, the sensitivity, $S=\frac{d V}{d x}$, as a function of $x$ is displayed under both analyzed configurations. The linear $S(x)$ behavior of the cylindrical permanent magnet reflects the non-linear response of $V(x)$ under this configuration. However, the rectangular magnet gives rise to a linear $V(x)$ response with nearly constant sensitivity for $2 \mathrm{~cm} \leq x \leq 5.5 \mathrm{~cm}$. Therefore, in spite of the lower maximum sensitivity under this rectangular magnet configuration, the enlargement of the sensor span (operational range) keeping constant sensitivity, could be considered as a key factor regarding sensing optimization and technical applications.

The differences in the sensor response can be analyzed in terms of the effective magnetic field acting on the sensing element, $H_{m}$, and the particular magnetic field gradient generated by each magnet, $\left|d H_{m} / d x\right|$. As fig. 3b shows, the cylindrical magnet displays a higher magnetic field gradient that in a first approximation could be associated to a higher sensor sensitivity. However, it should be kept in mind the non-linear characteristics of the magnetoimpedance voltage, that is, the non-linear $Z(H)$ behavior and the saturation of the GMI effect for large applied magnetic fields where $Z \approx R d c(d c$ resistance of the sample). This behavior is clearly reflected in the inset of fig. $3 \mathrm{~b}$, where $V_{L}=V / L$ (output voltage per unit length) is plotted as a function of a uniform magnetic field $H$ applied by a long solenoid along the wire axis $(L=3 \mathrm{~cm})$. Accordingly, the sharp decrease of $H_{m}$ with $x$ in the cylindrical magnet leads to a lower sensor operation range (sensor span) when compared with the rectangular configuration (as fig. 3a reflects). 

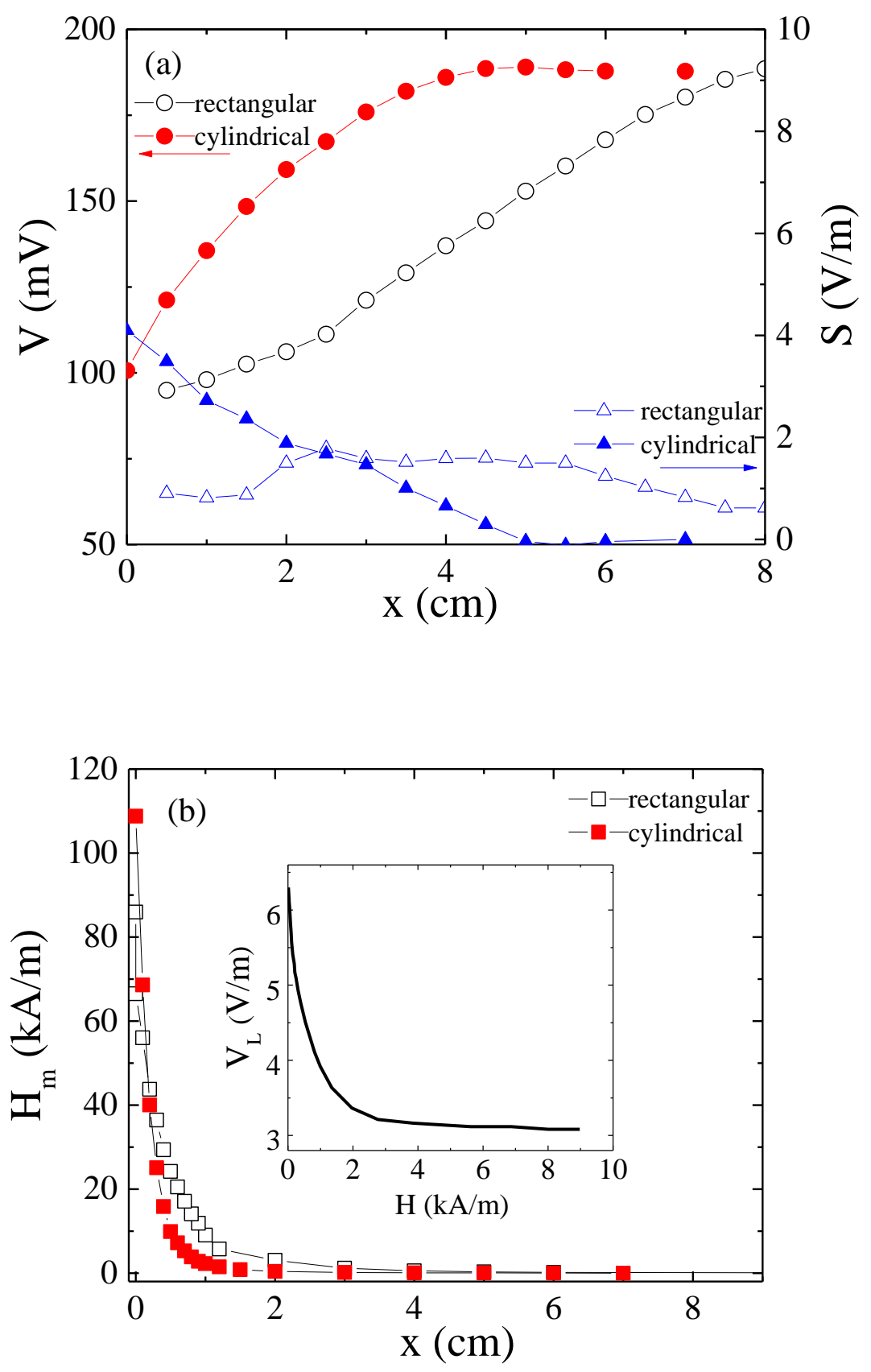

Fig. 3: (a) Sensor output voltage (circles), $V$, and sensitivity (triangles), $S$, as a function of the magnet position, $x$, under both permanent magnet configuration: (o) rectangular and ( $\bullet$ ) cylindrical; $(\triangle)$ rectangular and $(\Delta)$ cylindrical (b) Magnetic field generated by the magnet, $H_{m}$, versus the distance $x:(\square)$ rectangular and ( $\square$ ) cylindrical. Inset: Output voltage per unit length $V_{L}=V / L$, as a function of the axial homogenous magnetic field, $H$. 
However, the fact that $H_{m}$ is not strictly constant along the sample length should be taken into account in the analysis of the sensor response. In a first approximation, it can be roughly estimated assuming a homogenous magnetic field $H_{m}\left(x_{l}\right)$ corresponding to the closest point of the sensor to the permanent magnet, $x_{1}=x$ (see fig. 2) and considering the magnetoimpedance voltage at $H_{1}=H_{m}\left(x_{1}\right)$. Fig. 4 shows the estimated $V(x)$ under this first approximation $(n=1)$ where $V=V_{L}\left(H_{1}\right) L$, with $V_{L}=V / L$ the output voltage per unit length. It can be seen that the procedure roughly reproduces the experimental output voltage. In order to improve this estimation, the sensor length should be divided in $n$ segments and the voltage accordingly estimated:

$$
V(x)=\sum_{i=1}^{n} V_{L}\left(H_{i}\right) \frac{L}{n}
$$

with $H_{i}=H_{m}\left(x_{i}\right)$ and $x_{i}=x+\frac{i-1}{n} L$. Fig. 4 includes the results for $n=2$ and 4 . As expected, as $n$ increases the estimation procedure converges to the experimental response. However, no clear further improvement is obtained for larger values of $n$. This simple approximation (i.e. $n=4$ ) properly fits the experimental results for $x \leq 5 \mathrm{~cm}$, but it slightly departures for larger magnet distances. The wire's axis misalignment and the out of axis magnetic field components could contribute to the departure from the experimental response. Anyway, this simple estimation procedure is able to reproduce the basic features of $V(x)$ irrespectively of the actual magnetic field gradient and could be employed as a designing tool for non-contact position sensing devices. 


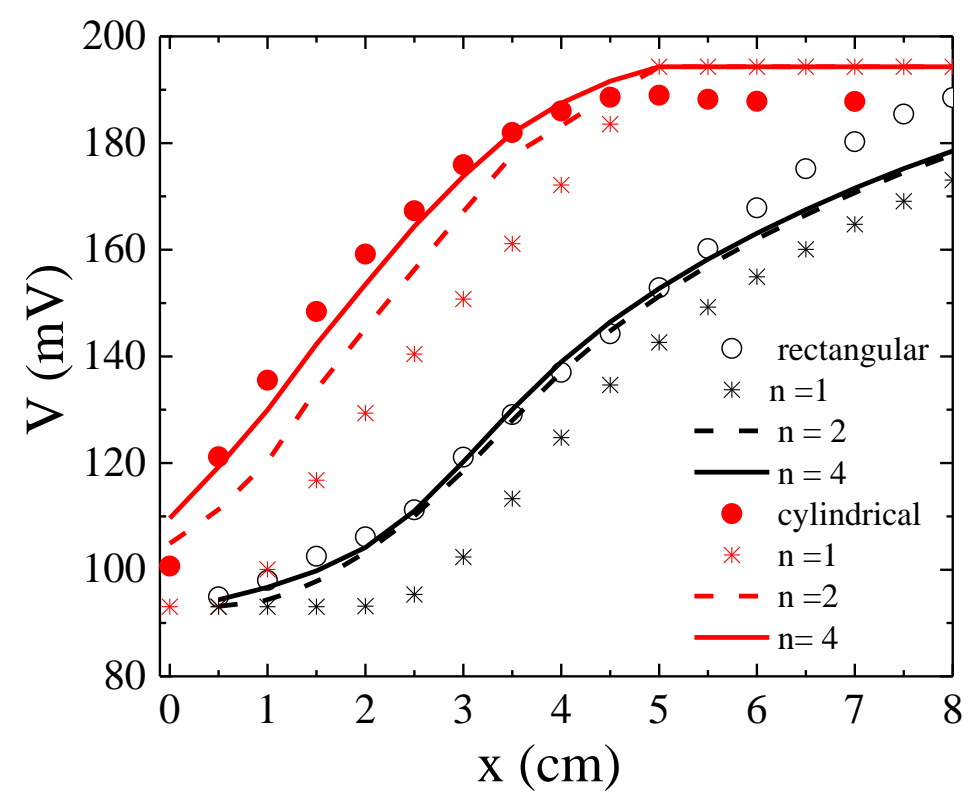

Fig. 4: Sensor output voltage, $V$, as a function of the magnet position, $\mathrm{x}$, under both permanent magnet configuration: (o) rectangular and (๑) cylindrical (experimental data); estimated output voltage: $\left(^{*}\right) n=1$, dashed line $n=2$, solid line $n=4$.

\subsection{Micrometric position characterization}

Regarding the characterization of the sensor response in the micrometric range, the rectangular permanent magnet was selected. As previously discussed, this magnet leads to a larger operational range with nearly constant sensitivity (see fig. 3a). Initially, the closest sensor end $(A)$ was placed at an initial position $x_{0} \approx 3 \mathrm{~cm}$ and $x$ was changed employing the micrometric screw $(10 \mu \mathrm{m})$. Fig. 5 shows the sensor response, $V(x)$, for the single sensor element $(L=3 \mathrm{~cm})$ and under the multicontact configuration $(L=12 \mathrm{~cm}$ and $L_{c}=3 \mathrm{~cm}$, see fig. 2). Both measurement configurations lead to a linear sensor response in the micrometric range with slight higher sensitivity, $S$, (slope) for the single $3 \mathrm{~cm}$ wire. In fact, similar linear $V(x)$ curves are obtained for different sample lengths under both configurations. Nevertheless, it is found that $\mathrm{S}$ clearly depends on $L$ and $L_{c}$. Fig. 6 shows the evolution of $S$ versus the effective sample length, $L_{e f}\left(L_{e f}=L\right.$ for the 
single wire and $L_{e f}=L_{c}$ for the multicontact configuration). The single wire displays slightly higher $S$ values for $L \leq 9 \mathrm{~cm}$, achieving maximum sensitivity for $L=7 \mathrm{~cm}$.

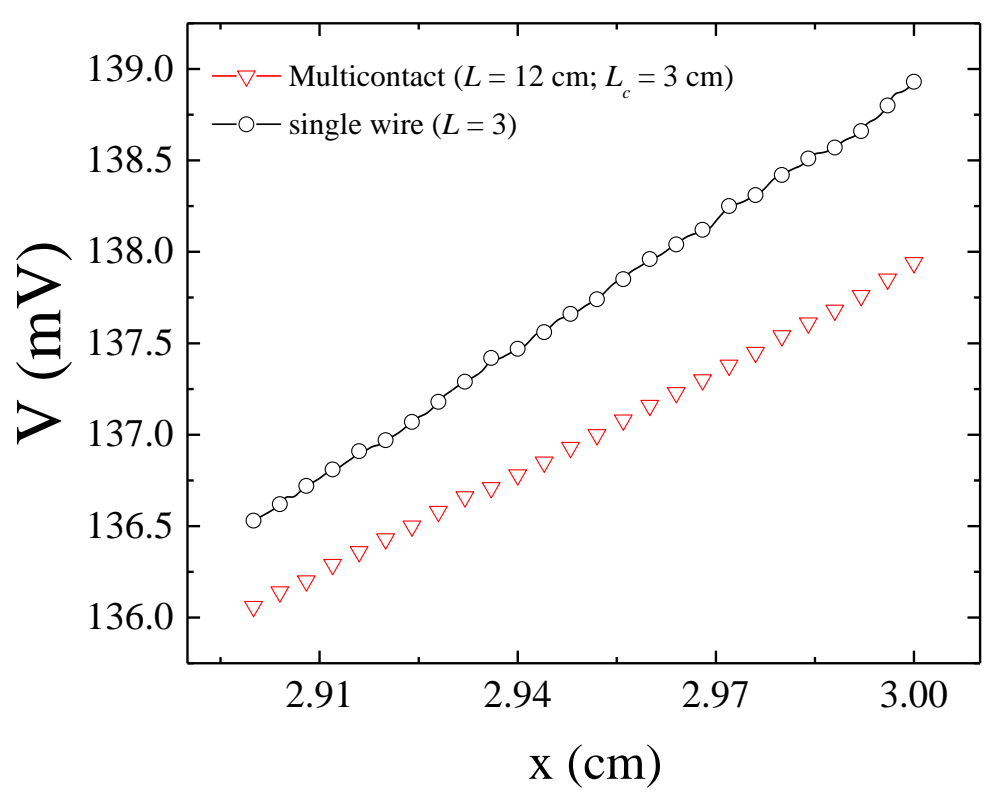

Fig. 5: Sensor output voltage, $V$, versus the magnet position, $x$, for (o) the single sensor element $(L=3 \mathrm{~cm})$ and $(\nabla)$ under the multicontact configuration $\left(L=12 \mathrm{~cm} ; L_{\mathrm{c}}=3 \mathrm{~cm}\right)$.

The best sensor performance of the single element in comparison with the multicontact configuration is also confirmed when $S$ is normalized to the sample length, $S_{L}=S / L$ (see inset of fig. 6). A monotonous decrease with $L_{e f}$ is observed, once more with higher values for the single wire, in this case for $L \leq 7 \mathrm{~cm}$. After that, both configurations converge to almost the same $S$ value. This behavior should be interpreted as a consequence of the domain structure and its effect on magnetoimpedance effect. Closure domain structure appears at the ends of the wire to reduce the magnetostatic energy, causing a hardening of the magnetic response in this sample end region [20-23]. This behavior arises as a result of the pinning of the domain walls at this sample ends, giving rise to an increase and decrease in the local coercivity and magnetic susceptibility, respectively. Thus, the decrease in $S$ for $L \leq 7 \mathrm{~cm}$ should be ascribed to the dominant role 
of the closure domains leading to a diminution in the magnetoimpedance ratio of the sample (see fig. 1b). Nevertheless, it can be concluded that sensor $7 \mathrm{~cm}$ in length shows the highest sensitivity because it displays the maximum effective length subjected to a region where magnetic field gradient exerts a relevant effect in terms of voltage variations. From $7 \mathrm{~cm}$ as length increases, a longer part of the sensor is out of the effect of the magnetic field gradient, leading to larger regions that do not contribute to the magnetoimpedance voltage variations. However, when single wire and multicontact configuration are compared, the highest sensitivity values are achieved in the single wires, where in principle it should be expected a lower magnetoimpedance response. For short samples the demagnetizing field becomes larger and more complex closure domain structures arise that would in principle lead to a decrease in the GMI response.

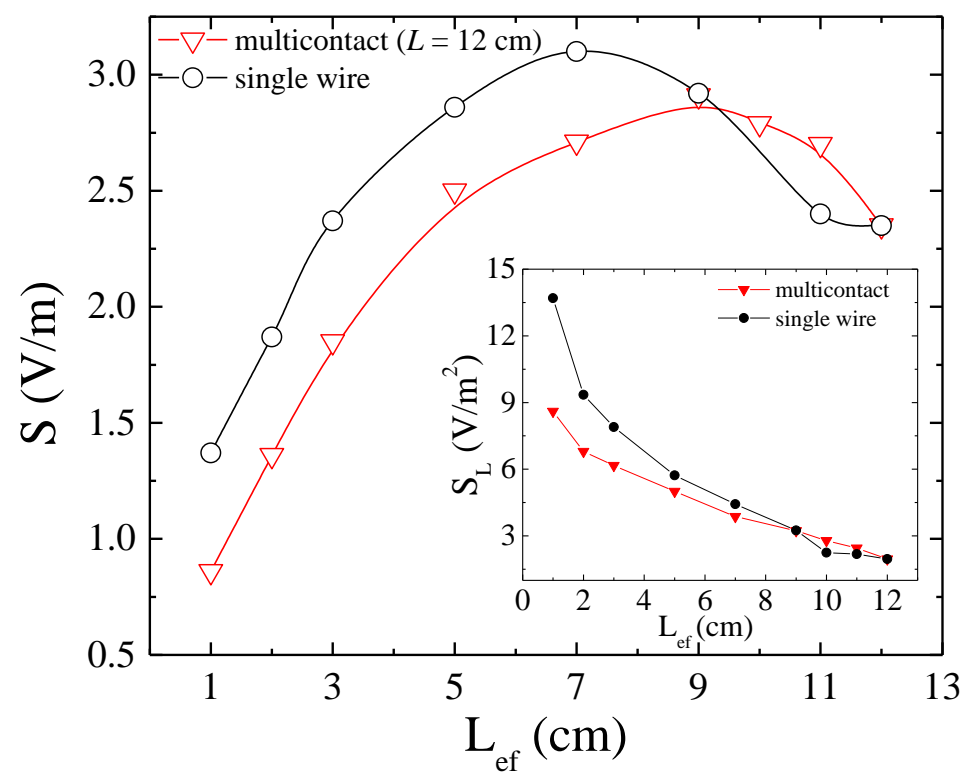

Fig. 6: Sensitivity, $S$, versus the effective length sensor length, $L_{e f}$, for the (o) single wire $\left(L_{e f},=L\right)$ and $(\nabla)$ under the multicontact configuration $\left(L=12 \mathrm{~cm} ; L_{e f},=L_{\mathrm{c}}\right)$. Inset: Sensitivity per unit length, $S_{L}$, vs $L_{e f}$.

To confirm the evolution of the magnetoimpedance response, the changes in the impedance, $Z$, at $x=3 \mathrm{~cm}$, as a function of $L_{e f}$ are displayed in fig.7. For comparison, the 
evolution of $Z$ under the multicontact configuration is also plotted, together with $R_{d c}$ versus $L_{e f}$. An almost linear increase in $\mathrm{Z}$ with $L_{e f}$ is found with a slightly higher slope for the single wires. Both linear responses in $Z$ and $R_{d c}$ can be understood in terms of classical electrodynamics ( $\mathrm{Z} \propto L$, eq. 1 and $R_{d c}=\frac{L}{\sigma \pi a^{2}}$ ), respectively. An equivalent GMI ratio can be defined as:

$$
\frac{\Delta Z_{m}}{Z}(\%)=\left(\frac{Z\left(L_{e f}\right)-R_{d c}\left(L_{e f}\right)}{R_{d c}\left(L_{e f}\right)}\right) \times 100
$$

where $Z\left(L_{e f}\right)$ corresponds to the impedance for the magnet position at $x=3 \mathrm{~cm}$ and $R_{d c}\left(L_{e f}\right)$ the $d c$ electrical resistance, both quantities evaluated for each $L_{e f}$. The inset of fig. 7 shows the dependence of $\frac{\Delta Z_{m}}{Z}$ as a function of $L_{e f}$ under both measuring configurations. The occurrence of an optimal length (namely, maximum GMI ratio) for single wire configuration is not observed as shown in fig. $1 \mathrm{~b}$ and reported in similar magnetoimpedance elements [24]. It should be kept in mind that $Z$ is determined under a non-uniform magnetic field acting on the samples and accordingly it could be expected a different evolution from the usually reported GMI evolution under uniform magnetic field. Anyway, a clear enhancement in $\frac{\Delta Z_{m}}{Z}$ ratio for the single wire elements is detected in comparison with the multicontact configuration for $L_{e f} \leq 5 \mathrm{~cm}$. Comparable results are obtained under uniform magnetic field in similar non-magnetostrictive amorphous wires. In fact, a clear improvement in the GMI ratio of a $\mathrm{FeCrSiBcuNb}$ wire is reported in single short elements in comparison with the impedance evolution measured for an equivalent partial of length on the longer samples [21]. As previously discussed, the particular domain structure associated to the demagnetizing field contribution plays a dominant role in the magnetoimpedance response of the wires. Although, its main contribution is to diminish both GMI ratio and $S$ as the effective length decreases, surprisingly it also promotes a slight enhancement in the magnetoimpedance response when comparing both 
configurations (single wire and multicontact) for the same $L_{e f}$. The occurrence of closure domains structures near the ends of non-magnetostrictive amorphous wires has been confirmed through the inspection of the magnetic susceptibility profile and magneto-optic Kerr effect along the samples [25]. For long wires, the susceptibility profile shows two peaks (closure domains) at both sample ends and a central plateau (domain walls at the central region of the wire). The reduction of the sample length gives rise to a collapse of both closure domain structures and a single peak susceptibility profile. Thus, the domain structure in a short wire and that at the sample end of a long wire with equivalent length are not strictly comparable. These changes in the domain structure and the expected modifications in the circular magnetic permeability should be the main origin of the detected differences in the magnetoimpedance response between both sensor configurations.

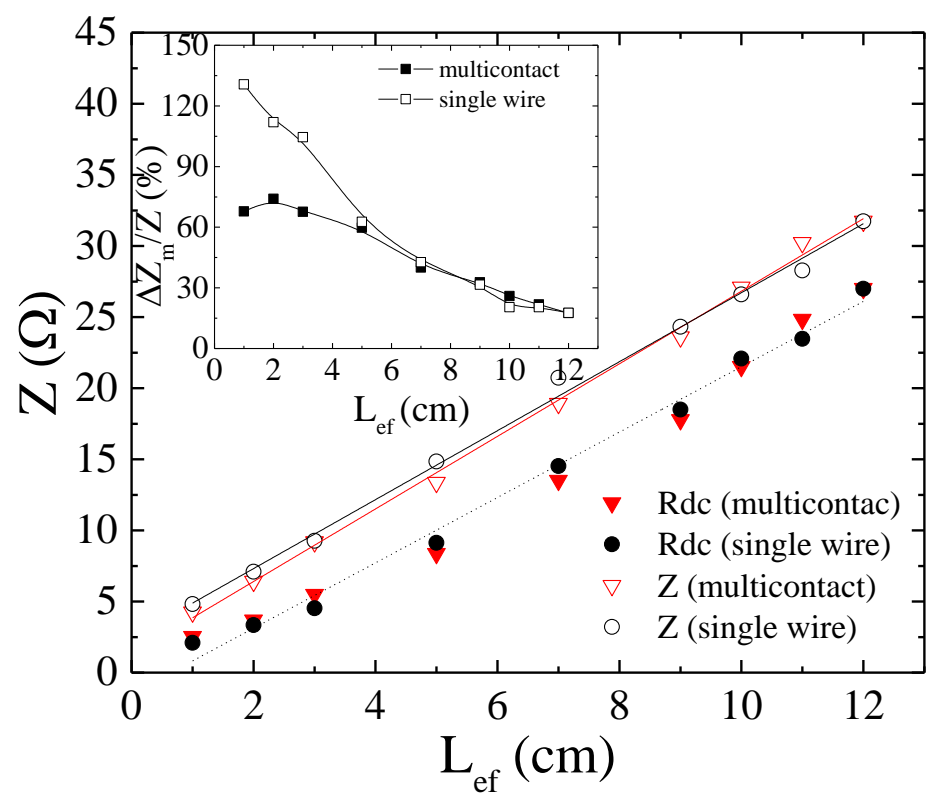

Fig. 7: Impedance, $Z$, at $x=3 \mathrm{~cm}$ (open symbols) and $d c$ electric resistance, $R_{d c}$, (closed symbols) as a function of the effective length sensor length, $L_{e f}$, for the (o) single wire $\left(L_{e f}=L\right)$ and $(\nabla)$ under the multicontact configuration $\left(L=12 \mathrm{~cm} ; L_{e f}=L_{\mathrm{c}}\right)$. Inset: GMI ratio $\frac{\Delta Z_{m}}{Z}(\%)$ vs $L_{e f}$. 


\subsection{Detection of micrometric trunk shrinkage}

Finally, on the basis of the previous analysis, a simple detector for the detection of the daily micrometric trunk shrinkage variation in plants was designed. As previously outlined, this type of detectors are of growing interest in the agronomic sector for irrigation scheduling purposes. Fig. 8a shows a photograph of the employed experimental device, where the rectangular magnet is fixed to the trunk of a lemon tree. The trunk shrinkage as a result of the water loss due to evaporation occurring during the illumination period, gives rise to an increase in $x$ and accordingly an increment in the sensor voltage. Conversely, the trunk growth during the darkness period has associated a decrease in $x$ and thus the diminution in the sensor voltage (increase in the effective magnetic field acting on the wire). According to the previous study, a wire $3 \mathrm{~cm}$ in length was selected as sensor nucleus. Such a wire's length has associated a relatively high micrometric sensitivity, and avoids misalignment problems associated to larger sample lengths. In order to avoid calibration problems due the plant fixing conditions (i.e. magnet axis misalignment due to irregular surface of the trunk), the sensor response was recalibrated once fixed in the plant and the sensitivity estimated within the mm range. Previous tests showed that the value of sensitivity within the millimeter range does not significantly change with regard the sensitivity measured within the micrometric range (employing the micrometric screw). Fig. $8 \mathrm{~b}$ displays the evolution of the sensor response for a three days cycle. For comparison, the trunk diameter changes measured employing a commercial LVDT sensor are also displayed. The inset clearly shows the linear relationship between both sensor signals (slope close to 1) along the analyzed time period. The results clearly show the possibility to accurately determine the micrometric trunk changes with this simple and low cost sensor. 

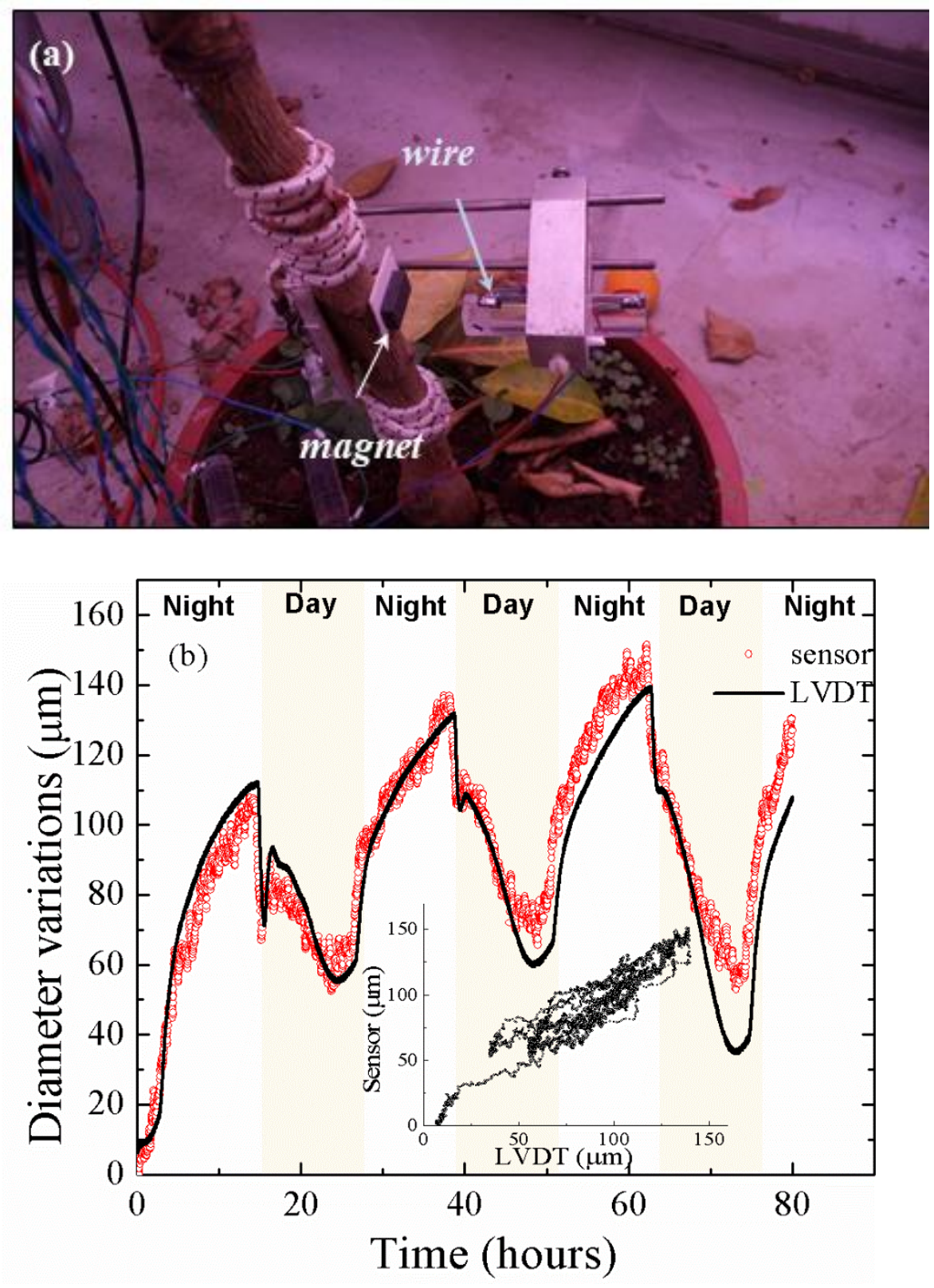

Fig. 8: (a) Non-contact position sensor fixed to the lemon tree; (b) Relative variations of the trunk diameter measured simultaneously by (-) the commercial LVDT and (o) the non-contact position sensor.

\section{Conclusions}

The analysis of the design of non-contact position technology based on the Giant Magnetoimpeance (GMI) effect is addressed employing a FeCo soft magnetic amorphous wire as sensing nucleus. The sensor is based on the changes of the high frequency electric impedance as a consequence of the effective magnetic field acting on it. In this non- 
contact position configuration a non-uniform magnetic field is generated by a permanent magnet whose relative position should be determined. The results show that optimum sensitivity is achieved for wires with a certain critical length, with a slight enhancement under a single wire configuration in comparison with the sensor response for a long wire with different voltage contacts along its axis. The complex closure domain structure at the sample ends is invoked as the main origin of the detected sensitivity enhancement. Finally, the analyzed position sensor is applied for the detection of the daily micrometric trunk shrinkage variation in plants for water monitoring purposes. The sensor, attached to a young lemon tree, is able to monitor the micrometric changes of the trunk diameter with equivalent sensitivity to a commercial LVDT. The results indicate that this type of magnetic sensors can be easily implemented in the agricultural sector, providing a low cost and sensitive detection technique.

Acknowledgements.- The work has been performed within the framework of the project VITICS, IIM14244.RI1 financially supported by the Departamento de Economía, Haciencia, Industria y Empleo, Navarra Government (Spain) and cofinanced with FEDER EU funds. The soft magnetic wire was kindly provided by Dr. A. Mitra, from NDE \& Magnetic Materials Group, CSIR-National Metallurgical Laboratory, Jamshedpur 831007, India. The authors would also like to acknowledge the assistance of Dr. L.G. Santesteban (Dept. Agricultural Production, UPNa) in the fitotron characterization.

\section{References}

1.- J.R. Brauer, Magnetic Actuators and Sensors, IEEE Press, Piscataway, 2014.

2.- J. Lenz, A.S. Edelstein, Magnetic sensors and their applications, IEEE Sens. J. 6 (2006) 631-649. 
3.- P. Ripka, Advances in magnetic field sensors, IEEE Sens. J. 10 (2010) 11081116.

4.- marketsandmarkets.com, Position Sensor Market by Type (Linear and Rotary), Contact Type (Contact and Non-Contact) Output (Analog and Digital), Application (Machine Tools, Robotics, Motion Systems, Material Handling), Industry, and Geography - Global Forecast to 2022.

5.- K. Mohri, T. Uchiyama, L.P. Shen, C.M. Cai, L.V. Panina, Amorphous wire and CMOS IC-based sensitive micro-magnetic sensors (MI sensor and SI sensor) for intelligent measurements and controls, J. Magn. Magn. Mat. 249 (2002) 351-356.

6.- A. García-Arribas, J. Gutierrez, G.V. Kurlyansdskaya, J.M. Barandiarán, A. Svalov, E. Fernández, A. Lasheras, D. de Cos, I. Bravo-Imaz, Sensor applications of soft magnetic materials based-on magneto-impedance, magneto-elastic resonance and magneto-electricity, Sensors 14 (2014) 7602-7624.

7.- A. Diaz-Rubio, H. García-Miquel, V.M. García-Chocano, In-plane omnidirectional magnetic field sensor based on Giant Magneto Impedance (GMI), J. Magn. Magn. Mat. 444 (2017) 249-255.

8.- M. Knobel, M. Vázquez, L. Kraus, Giant Magnetoimpedance, in Handbook of Magnetic Materials, vol. 15 (2003) 497-563.

9.- L.D. Landau, E.M. Lifschitz, L.P. Pitaevskii, Electrodynamics of continuous Media (Butterworth-Heinemann, London, 1995), p. 212.

10.- K. Mohri, T. Kohzawa, T. H. Yoshida, L.V. Panina, Magneto-Inductive effect (MI effect) in amorphous wires, IEEE Trans. Mag. 28 (1992) 3150-3152.

11.- J. Velázquez, M. Vázquez, D.-X. Chen, A. Hernando, Giant magnetoimpedance in nonmagnetostrictive amorphous wires, Phys. Rev. B 50 (1994) 16737-16740.

12.- M.-H. Phan, H.-X. Peng, Giant magnetoimpedance materials: Fundamentals and applications, Prog. Mater. Sci. 53 (2008) 323-420.

13.- M. Han, D.F. Liang, L.J. Denag, Sensor development using its unusual properties of Fe/co-based amorphous soft magnetic wire, J. Mater. Sci. 40 (2005) 55735580.

14.- R. Valenzuela, m. Vázquez, A. Hernando, A position sensor base don magnetoimpedance, J. Appl. Phys. 79 (1996) 6549-6551. 
15.- F.E. Atalay, S. Atalay, A position sensor based on magnetoimpedance effect, Phys. Status Solidi A-Appl. Mat 189 (2002) 311-315.

16.- M. Ipatov, V. Zhukova, J.M. Blanco, A. Zhukov, J. González, $1 D$ and 2D position detection using magentoimpedance sensor array, Phys. Status Solidi A-Appl. Mat 209 (2012) 2626-2629.

17.- D.A. Goldhamer, E. Fereres, Irrigation scheduling protocols using continuously recorded trunk diameter measurements, Irrig. Sci. 20 (2001) 115-125.

18.- J.E. Fernández, M.V. Cuevas, Irrigation scheduling from stem diameter variations: A review, Agric. For. Meteorol. 150 (2010) 135-151

19.- T.K. Das, A. Mitra, S.K. Mandal, R.K. Roy, P. Banerji, A.K. Panda, Parametric control son giant magnetoimpedance (GMI) behaviour of CoFeSiBCr amorphous wires for prospective sensor applications, Sens. Actuator A-Phys. 220 (2014) 382-387.

20.-T. Reininger, H. Krönmuller, C. Gómez-Polo, M. Vázquez, Magnetic domain observation in amorphous wires, J. App. Phys. 73 (1993) 5357-5359.

21.- M. Vázquez, Y.-F. Li and D.-X. Chen, Influence if the sample length and profile of the magnetoimpedance effect in FeCrSiBCuNb ultrasoft magnetic wires, J. Appl. Phys. 91 (2002) 6539.

23.- V. Zhukova, N.A. Usov, A. Zhukov, J. González, Length effect in a Co-rich amorphous wire, Phys. Rev. B. 65, (2002) 134407.

24.-A. Chaturvedi, T. Dhakal, S. Witanachchi, A. Le, M. Phan, H. Srikanth, Critical length and giant magnetoimpedance in $\mathrm{Co}_{69} \mathrm{Fe}_{4.5} \mathrm{Ni}_{1.5} \mathrm{Si}_{10} \mathrm{~B}_{15}$ amorphous ribbons, Mater. Sci. Eng. B-Solid State Mater. 172 (2010) 146-150.

25.- M. Vázquez, H. Theuss, H. Kronmüller, Susceptibility profile in soft magnetic wires, IEEE Trans. Mag. 35 (1999) 573-579. 


\section{Figure Captions}

Fig. 1: (a) GMI ratio as a function of the exciting frequency, $f$, (current amplitude, $I_{p p}=15 \mathrm{~mA}$ ) and versus $I_{p p}$ (inset) for a wire $3 \mathrm{~cm}$ in length. (b) GMI ratio as a function of the sample length, $L$ (frequency $f=300 \mathrm{kHz}$ and current amplitude, $I_{p p}=15 \mathrm{~mA}$ ).

Fig. 2: Sensor configuration schedule

Fig. 3: (a) Sensor output voltage (circles), $V$, and sensitivity (triangles), $S$, as a function of the magnet position, $x$, under both permanent magnet configuration: (o) rectangular and $(\bullet)$ cylindrical; $(\triangle)$ rectangular and $(\boldsymbol{\Delta})$ cylindrical (b) Magnetic field generated by the magnet, $H_{m}$, versus the distance $x:(\square)$ rectangular and ( $\square$ ) cylindrical. Inset: Output voltage per unit length $V_{L}=V / L$, as a function of the axial homogenous magnetic field, $H$.

Fig. 4: Sensor output voltage, $V$, as a function of the magnet position, $\mathrm{x}$, under both permanent magnet configuration: (o) rectangular and (๑) cylindrical (experimental data); estimated output voltage: $(*) n=1$, dashed line $n=2$, solid line $n=4$.

Fig. 5: Sensor output voltage, $V$, versus the magnet position, $x$, for (o) the single sensor element $(L=3 \mathrm{~cm})$ and $(\nabla)$ under the multicontact configuration $\left(L=12 \mathrm{~cm} ; L_{\mathrm{c}}\right.$ $=3 \mathrm{~cm})$.

Fig. 6: Sensitivity, $S$, versus the effective length sensor length, $L_{e f}$, for the (o) single wire $\left(L_{e f},=L\right)$ and $(\nabla)$ under the multicontact configuration $\left(L=12 \mathrm{~cm} ; L_{e f}=L_{\mathrm{c}}\right)$. Inset: Sensitivity per unit length, $S_{L}$, vs $L_{e f}$.

Fig. 7: Impedance, $Z$, at $x=3 \mathrm{~cm}$ (open symbols) and $d c$ electric resistance, $R_{d c}$, (closed symbols) as a function of the effective length sensor length, $L_{e f}$, for the (o) single wire $\left(L_{e f},=L\right)$ and $(\nabla)$ under the multicontact configuration $\left(L=12 \mathrm{~cm} ; L_{e f},=L_{\mathcal{c}}\right)$. Inset: GMI ratio $\frac{\Delta Z_{m}}{Z}(\%)$ vs $L_{e f}$. 
Fig. 8: (a) Non-contact position sensor fixed to the lemon tree; (b) Relative variations of the trunk diameter measured simultaneously by (-) the commercial LVDT and (o) the non-contact position sensor. 